\title{
NONLINEAR WAVE EQUATIONS AND REACTION-DIFFUSION EQUATIONS WITH SEVERAL NONLINEAR SOURCE TERMS OF DIFFERENT SIGNS AT HIGH ENERGY LEVEL
}

\author{
RUNZHANG XU ${ }^{\otimes 1}$, YANBING YANG ${ }^{2}, \mathrm{SHAOHUA} \mathrm{CHEN}^{3}, \mathrm{JIA} \mathrm{SU}^{4}$, \\ JIHONG SHEN $^{1}$ and SHAOBIN HUANG ${ }^{5}$
}

(Received 28 November, 2012; revised 31 December, 2012; first published online 11 June, 2013)

\begin{abstract}
This paper is concerned with the initial boundary value problem of a class of nonlinear wave equations and reaction-diffusion equations with several nonlinear source terms of different signs. For the initial boundary value problem of the nonlinear wave equations, we derive a blow up result for certain initial data with arbitrary positive initial energy. For the initial boundary value problem of the nonlinear reaction-diffusion equations, we discuss some probabilities of the existence and nonexistence of global solutions and give some sufficient conditions for the global and nonglobal existence of solutions at high initial energy level by employing the comparison principle and variational methods.
\end{abstract}

2010 Mathematics subject classification: 35L05.

Keywords and phrases: wave equation, reaction-diffusion equation, high energy level, finite time blow-up, variational method, comparison principle.

\section{Introduction}

In this paper, we consider the initial boundary value problem (IBVP) of the wave equation with several nonlinear source terms of different signs,

$$
\begin{gathered}
u_{t t}-\Delta u=f(u) \equiv \sum_{k=1}^{l} a_{k}|u|^{p_{k}-1} u-\sum_{j=1}^{s} b_{j}|u|^{q_{j}-1} u, \quad x \in \Omega, t>0, \\
u(x, t)=0, \quad x \in \partial \Omega, t \geq 0, \\
u(x, 0)=u_{0}(x), \quad u_{t}(x, 0)=u_{1}(x), \quad x \in \Omega ;
\end{gathered}
$$

\footnotetext{
${ }^{1}$ College of Science, Harbin Engineering University, Harbin 150001, PR China; e-mail: xurunzh@yahoo.com.cn.

${ }^{2}$ College of Automation, Harbin Engineering University, Harbin 150001, PR China.

${ }^{3}$ Department of Mathematics, Cape Breton University, Sydney, NS, Canada B1P 6L2.

${ }^{4}$ Science China Press, Beijing 100717, PR China.

${ }^{5}$ College of Computer Science and Technology, Harbin Engineering University, Harbin 150001, PR

China.

(C) Australian Mathematical Society 2013, Serial-fee code 1446-1811/2013\$16.00
} 
and the IBVP of reaction-diffusion equations with several nonlinear source terms of different signs,

$$
\begin{gathered}
u_{t}-\Delta u=f(u) \equiv \sum_{k=1}^{l} a_{k}|u|^{p_{k}-1} u-\sum_{j=1}^{s} b_{j}|u|^{q_{j}-1} u, \quad x \in \Omega, t>0, \\
u(x, 0)=u_{0}(x), \quad x \in \Omega, \\
u(x, t)=0, \quad x \in \partial \Omega, t \geq 0 .
\end{gathered}
$$

Here $\Omega \subset \mathbb{R}^{n}(n \geq 1)$ is a bounded domain with smooth boundary $\partial \Omega$ and $a_{k}>0$, $p_{k}>1,1 \leq k \leq l, b_{j}>0, q_{j}>1,1 \leq j \leq s$. Furthermore, $p_{k}$ and $q_{j}$ satisfy

$$
\begin{cases}1<q_{s}<q_{s-1}<\cdots<q_{1}=q<p=p_{l}<p_{l-1}<\cdots<p_{1}<\infty & \text { if } n=1,2, \\ 1<q_{s}<q_{s-1}<\cdots<q_{1}=q<p=p_{l}<p_{l-1}<\cdots<p_{1} \leq \frac{n+2}{n-2} & \text { if } n \geq 3 .\end{cases}
$$

This paper continues the work done by Liu and $\mathrm{Xu}$ [7], so we begin with just a quick introduction. We refer the reader to the paper by Liu and $\mathrm{Xu}$ [7] and the references therein for background. The motivation for studying such combined power type nonlinearities can also be found in papers by Li and Zhang [4], Tao et al. [10] and Vuillermot [11]. Liu and $\mathrm{Xu}$ [7] obtained both the global and nonglobal existence of solutions for problems (1.1)-(1.3) and (1.4)-(1.6) for the case $E(0)<d$, where $E(0)$ is the initial energy and $d$ is the depth of the potential well that will be given later. They only proved the global existence of solutions for problem (1.1)-(1.3) in the critical case $E(0)=d$. For this critical case, the nonexistence of global solutions for problem (1.1)-(1.3) and the global existence of solutions for problem (1.4)-(1.6) were given by Yu et al. [12]. However, the depth of the potential well $d$ for problems (1.1)-(1.3) and (1.4)-(1.6) is usually very small, which means that the initial conditions mostly do not satisfy the high energy case $E(0)>d$. To our knowledge, the global well-posedness of solutions for problems (1.1)-(1.3) and (1.4)-(1.6) at the high initial energy level $E(0)>d$ is an open problem, and it is tackled in this paper.

Wave equation. In the low initial energy case $E(0)<d$, it is required to prove the invariance of the unstable set under the flow of problem (1.1)-(1.3) by contradiction in advance. This plays an important role in proving the finite time blow-up of solutions with arbitrary positive initial energy. In other words, we should deduce that $J\left(u\left(t_{0}\right)\right) \geq d$, which is contradictory to $J\left(u\left(t_{0}\right)\right) \leq E(0)<d$. Under the condition $E(0)>d$, this contradiction is not established, which is the biggest difficulty we face. Thus it is worth investigating the qualitative properties of solutions for problem (1.1)(1.3) in the case of $E(0)>d$. By utilizing the technique of Gazzola and Squassina [2] and the so-called concavity method $[5,6]$, this paper obtains the invariant unstable set and derives a sufficient condition on the initial data with arbitrary positive initial energy such that the corresponding local solution of problem (1.1)-(1.3) blows up in finite time.

Reaction-diffusion equation. Although Gazzola and Weth [3] have obtained the global existence and finite time blow-up of solutions for problem (1.4)-(1.6) 
with $f(u)=|u|^{p-1} u$ at high initial energy level $J\left(u_{0}\right)>d$ by the comparison principle and variational methods, we find that there is only one source term in their problem and the sign of that source term $\left(f(u)=|u|^{p-1} u\right)$ is the same as the sign of $u$. In addition, for problem (1.4)-(1.6) with several nonlinear source terms of different signs, in the high initial energy case $J\left(u_{0}\right)>d$ we cannot find the corresponding comparison principle, which plays a crucial role in proving the global existence of solutions for problem (1.4)-(1.6) by employing the method of Gazzola and Weth [3]. Hence it is of great meaning to solve this open problem. In this paper, by employing the strong maximum principle we obtain the comparison principle of problem (1.4)-(1.6). Then, by using this comparison principle together with variational methods, we prove finite time blowup and obtain global solutions for problem (1.4)-(1.6) for the high initial energy case $J\left(u_{0}\right)>d$.

The paper is organized as follows. The blow-up result of solutions with arbitrary positive initial energy for problem (1.1)-(1.3) is proved in Section 2. In Section 3 we determine for which initial data $u_{0}$ in the phase space $H_{0}^{1}(\Omega)$ the solution of problem (1.4)-(1.6) blows up and for which initial data $u_{0}$ the solution is globally defined at the high initial energy level $J\left(u_{0}\right)>d$.

Throughout the present paper, the following notation is used for precise statements: $L^{p}(\Omega)(2 \leq p<\infty)$ denotes the usual space of all $L^{p}$-functions on $\Omega$ with norm

$$
\|u\|_{p}=\|u\|_{L^{p}(\Omega)}, \quad\|u\|=\|u\|_{L^{2}(\Omega)},
$$

and inner product

$$
(u, v)=\int_{\Omega} u v d x
$$

\section{Wave equation: high energy blow-up}

In order to obtain the blow-up result of solutions with arbitrary positive initial energy for problem (1.1)-(1.3), we first introduce the energy functional

$$
E(t) \equiv E(u)=\frac{1}{2}\left\|u_{t}\right\|^{2}+\frac{1}{2}\|\nabla u\|^{2}-\sum_{k=1}^{l} \frac{a_{k}}{p_{k}+1}\|u\|_{p_{k}+1}^{p_{k}+1}+\sum_{j=1}^{s} \frac{b_{j}}{q_{j}+1}\|u\|_{q_{j}+1}^{q_{j}+1},
$$

the Nehari functional

$$
I(t) \equiv I(u)=\|\nabla u\|^{2}-\sum_{k=1}^{l} a_{k}\|u\|_{p_{k}+1}^{p_{k}+1}+\sum_{j=1}^{s} b_{j}\|u\|_{q_{j}+1}^{q_{j}+1}
$$

and the unstable set

$$
V=\left\{u \in H_{0}^{1}(\Omega) \mid I(u)<0\right\} .
$$

Next we give a definition of the weak solution for problem (1.1)-(1.3).

Definition 2.1. We say that $u=u(x, t)$ is a weak solution of problem (1.1)-(1.3) on $\Omega \times[0, T)$ if $u \in L^{\infty}\left(0, T ; H_{0}^{1}(\Omega)\right), u_{t} \in L^{\infty}\left(0, T ; L^{2}(\Omega)\right)$ and 
(i) $\left(u_{t}, v\right)+\int_{0}^{t}(\nabla u, \nabla v) d \tau=\int_{0}^{t}(f(u), v) d \tau+\left(u_{1}, v\right)$ for all $v \in H_{0}^{1}(\Omega), t \in(0, T)$;

(ii) $u(x, 0)=u_{0}(x)$ in $H_{0}^{1}(\Omega), u_{t}(x, 0)=u_{1}(x)$ in $L^{2}(\Omega)$;

(iii) $E(t)=E(0), t \in[0, T)$.

Definition 2.1 implies that if $u$ is a weak solution of problem (1.1)-(1.3) on $\Omega \times[0, T)$, then $u_{t t} \in L^{\infty}\left(0, T ; H^{-1}(\Omega)\right)$ and

$$
\left\langle u_{t t}, v\right\rangle=(f(u), v)-(\nabla u, \nabla v) \quad \text { for all } v \in H_{0}^{1}(\Omega), t \in(0, T),
$$

where $\langle\cdot, \cdot\rangle$ denotes the standard pairing of $H^{-1}(\Omega)$ and $H_{0}^{1}(\Omega)$.

In order to obtain the invariance of the unstable set $V$ under the flow of problem (1.1)-(1.3), which plays an important role in obtaining the global nonexistence of the weak solution for problem (1.1)-(1.3), we prove the following lemma in advance.

Lemma 2.2. Assume that $u_{0}(x) \in H_{0}^{1}(\Omega), u_{1}(x) \in L^{2}(\Omega)$ and let $u(x, t)$ be the weak solution of equation (1.1) with initial data $\left(u_{0}, u_{1}\right)$. Assume that the initial data satisfy

$$
\int_{\Omega} u_{0} u_{1} d x \geq 0
$$

Then the map $t \mapsto\|u(t)\|^{2}$ is strictly increasing as long as $u(t) \in V$.

Proof. Let

$$
F(t)=\|u(t)\|^{2}
$$

Then

$$
F^{\prime}(t)=2\left(u, u_{t}\right)
$$

Moreover, by using (2.3) and

$$
\left\langle u_{t t}(t), u(t)\right\rangle=\frac{d}{d t}\left(u, u_{t}\right)-\left\|u_{t}(t)\right\|^{2} \quad \text { for almost all } t \in[0, \infty),
$$

we have

$$
F^{\prime \prime}(t)=2\left\langle u_{t t}, u\right\rangle+2\left\|u_{t}\right\|^{2} .
$$

Notice that by testing equation (1.1) with $u$ we get

$$
\left\langle u_{t t}, u\right\rangle+\|\nabla u\|^{2}=\sum_{k=1}^{l} a_{k}\|u\|_{p_{k}+1}^{p_{k}+1}-\sum_{j=1}^{s} b_{j}\|u\|_{q_{j}+1}^{q_{j}+1} \quad \text { for } t \in[0, \infty) .
$$

Then equation (2.7) gives

$$
F^{\prime \prime}(t)=2\left\|u_{t}\right\|^{2}-2 I(u)
$$

Furthermore, from $u(t) \in V$,

$$
F^{\prime \prime}(t)>0 \text { for } t \in\left[0, T_{\max }\right) .
$$

By inequality (2.4), we have $F^{\prime}(0)=\int_{\Omega} u_{0} u_{1} d x \geq 0$. We see that $F^{\prime}(t)>F^{\prime}(0) \geq 0$, namely $F^{\prime}(t)>0$. Therefore, the map $t \mapsto\|u(t)\|^{2}$ is strictly increasing. 
In the following, we show the invariance of the unstable set under the flow of problem (1.1)-(1.3).

Lemma 2.3. Suppose that $u_{0}(x) \in H_{0}^{1}(\Omega), u_{1}(x) \in L^{2}(\Omega)$, and that assumption (1.7) and inequality (2.4) hold. Assume that the initial data satisfy

$$
\left\|u_{0}\right\|^{2}>\alpha E(0)
$$

where $\alpha=(2 / C)(1+2 /(p-1))$ and $C$ is the coefficient of the Poincaré inequality

$$
\|\nabla u\|^{2} \geq C\|u\|^{2} .
$$

Then all solutions of problem (1.1)-(1.3) with $E(0)>0$ belong to $V$, provided $u_{0} \in V$.

Proof. We prove that $u(t) \in V$. If this is false, let $t_{0} \in(0, T)$ be the first time such that $I(u(t))=0$, that is, $I(u(t))<0$ for $t \in\left[0, t_{0}\right)$ and $I\left(u\left(t_{0}\right)\right)=0$. Now let $F(t)$ be defined by (2.5) above. Hence from Lemma 2.2, we obtain that $F(t)$ and $F^{\prime}(t)$ are strictly increasing on the interval $\left[0, t_{0}\right)$. Then by inequality (2.9),

$$
F(t)>\left\|u_{0}\right\|^{2}>\alpha E(0) \text { for all } t \in\left[0, t_{0}\right) .
$$

Moreover, from the continuity of $u(t)$ in $t$,

$$
F\left(t_{0}\right)>\alpha E(0) .
$$

On the other hand, by equations (2.1) and (2.2) and assumption (1.7), we obtain

$$
\begin{aligned}
E(0)=E\left(t_{0}\right) & \geq \frac{1}{2}\left\|\nabla u\left(t_{0}\right)\right\|^{2}-\sum_{k=1}^{l} \frac{a_{k}}{p_{k}+1}\left\|u\left(t_{0}\right)\right\|_{p_{k}+1}^{p_{k}+1}+\sum_{j=1}^{s} \frac{b_{j}}{q_{j}+1}\left\|u\left(t_{0}\right)\right\|_{q_{j}+1}^{q_{j}+1} \\
& \geq \frac{1}{2}\left\|\nabla u\left(t_{0}\right)\right\|^{2}-\frac{1}{p+1}\left(\sum_{k=1}^{l} a_{k}\left\|u\left(t_{0}\right)\right\|_{p_{k}+1}^{p_{k}+1}-\sum_{j=1}^{s} b_{j}\left\|u\left(t_{0}\right)\right\|_{q_{j}+1}^{q_{j}+1}\right) \\
& =\left(\frac{1}{2}-\frac{1}{p+1}\right)\left\|\nabla u\left(t_{0}\right)\right\|^{2}+\frac{1}{p+1} I\left(u\left(t_{0}\right)\right) .
\end{aligned}
$$

Note that $I\left(u\left(t_{0}\right)\right)=0$. Hence

$$
\left\|\nabla u\left(t_{0}\right)\right\|^{2} \leq 2\left(1+\frac{2}{p-1}\right) E(0)
$$

Using the Poincaré inequality

$$
\|\nabla u\|^{2} \geq C\|u\|^{2}
$$

we obtain from inequality (2.11) that

$$
F\left(t_{0}\right)=\left\|u\left(t_{0}\right)\right\|^{2} \leq \frac{1}{C}\left\|\nabla u\left(t_{0}\right)\right\|^{2} \leq \frac{2}{C}\left(1+\frac{2}{p-1}\right) E(0)=\alpha E(0) .
$$

Inequality (2.12) contradicts inequality (2.10). 
We now present the main blow-up theorem for the weak solution with arbitrary positive initial energy.

THEOREM 2.4. Let $u_{0}(x) \in H_{0}^{1}(\Omega), u_{1}(x) \in L^{2}(\Omega)$ be given and assume that assumption (1.7) and inequalities (2.4) and (2.9) hold. Assume that $E(0)>0$ and $u_{0} \in V$. Then the weak solution of problem (1.1)-(1.3) blows up in finite time.

Proof. Let $u(t)$ be any weak solution of problem (1.1)-(1.3) with $E(0)>0$ and $u_{0} \in V$. Then from Lemma 2.3 we have $u(t) \in V$. Next, we prove that the weak solution of problem (1.1)-(1.3) blows up in finite time. Suppose by contradiction that the solution $u(x, t)$ is global. Then for any $T_{0}>0$, we define the auxiliary function $F(t)$ by (2.5).

We have $F(t)>0$ for all $t \in\left[0, T_{0}\right]$. From the continuity of $F(t)$ in $t$, there exists $\rho>0$ (independent of the choice of $T_{0}$ ) such that

$$
F(t) \geq \rho \quad \text { for all } t \in\left[0, T_{0}\right]
$$

Moreover, for $t \in\left[0, T_{0}\right]$, we obtain equations (2.6) and (2.8). From (2.6), by the Schwarz inequality,

$$
F^{\prime}(t)^{2}=4\left(u, u_{t}\right)^{2} \leq 4\|u\|^{2}\left\|u_{t}\right\|^{2}=4 F(t)\left\|u_{t}\right\|^{2} .
$$

Hence from inequality (2.14) and equation (2.8),

$$
\begin{aligned}
F^{\prime \prime}(t) F(t)-\frac{p+3}{4} F^{\prime}(t)^{2} & \geq F(t)\left(F^{\prime \prime}(t)-(p+3)\left\|u_{t}\right\|^{2}\right) \\
& \geq F(t)\left(2\left\|u_{t}\right\|^{2}-2 I(u)-(p+3)\left\|u_{t}\right\|^{2}\right) .
\end{aligned}
$$

Now define

$$
\xi(t)=-(p+1)\left\|u_{t}\right\|^{2}-2 I(u)
$$

Note that

$$
E(t)=E(0)
$$

Then by equations (2.1) and (2.2) and assumption (1.7),

$$
E(0) \geq \frac{1}{2}\left\|u_{t}\right\|^{2}+\left(\frac{1}{2}-\frac{1}{p+1}\right)\|\nabla u\|^{2}+\frac{1}{p+1} I(u) .
$$

Substituting (2.16) into (2.15), we deduce that

$$
\xi(t) \geq(p-1)\|\nabla u\|^{2}-2(p+1) E(0) .
$$

Let

$$
\theta(t)=(p-1)\|\nabla u\|^{2}-2(p+1) E(0) .
$$

Then from Lemma 2.2 and the Poincaré inequality, we conclude that

$$
\frac{2}{C}\left(1+\frac{2}{p-1}\right) E(0)<\left\|u_{0}\right\|^{2}<\|u\|^{2}<\frac{1}{C}\|\nabla u\|^{2} .
$$


By inequality (2.17), we have $\theta(t)>0$, that is, $\xi(t)>\sigma>0$. Therefore,

$$
F^{\prime \prime}(t) F(t)-\frac{p+3}{4} F^{\prime}(t)^{2} \geq \rho \sigma>0, \quad t \in\left[0, T_{0}\right] .
$$

Setting $y(t)=F(t)^{-(p-1) / 4}$, this inequality together with (2.13) gives

$$
y^{\prime \prime}(t) \leq-\frac{p-1}{4} \frac{\sigma}{\rho} y(t), \quad t \in\left[0, T_{0}\right] .
$$

This proves that $y(t)$ reaches 0 in finite time, say $t \rightarrow T_{*}$. Since $T_{*}$ is independent of the initial choice of $T_{0}$, we may assume that $T_{*}<T_{0}$. This tells us that

$$
\lim _{t \rightarrow T_{*}} F(t)=+\infty
$$

This completes the proof.

\section{Reaction-diffusion: high energy global well-posedness}

3.1. Set-up and notations In order to obtain the global existence and blow-up of solutions for problem (1.4)-(1.6) at high initial energy level, we first define the cone of nonnegative functions

$$
\mathbb{K}=\left\{u \in H_{0}^{1}(\Omega) \mid u \geq 0 \text { almost everywhere in } \Omega\right\} .
$$

Given any $u \in H_{0}^{1}(\Omega)$, we denote its positive part by

$$
u^{+}(x):=\max \{u(x), 0\}
$$

and its negative part by

$$
u^{-}(x):=\min \{u(x), 0\} .
$$

For problem (1.4)-(1.6) we also define the energy functional $J(u)$ and the Nehari functional $I(u)$ :

$$
\begin{aligned}
& J(u)=\frac{1}{2}\|\nabla u\|^{2}-\sum_{k=1}^{l} \frac{a_{k}}{p_{k}+1}\|u\|_{p_{k}+1}^{p_{k}+1}+\sum_{j=1}^{s} \frac{b_{j}}{q_{j}+1}\|u\|_{q_{j}+1}^{q_{j}+1}, \\
& I(u)=\|\nabla u\|^{2}-\sum_{k=1}^{l} a_{k}\|u\|_{p_{k}+1}^{p_{k}+1}+\sum_{j=1}^{s} b_{j}\|u\|_{q_{j}+1}^{q_{j}+1} .
\end{aligned}
$$

Since the Nehari mainfold plays a crucial role, we define

$$
\mathcal{N}=\left\{u \in H_{0}^{1}(\Omega) \backslash\{0\} \mid I(u)=0\right\},
$$

which separates the two unbounded sets

$$
\mathcal{N}_{+}=\left\{u \in H_{0}^{1}(\Omega) \mid I(u)>0\right\} \quad \text { and } \quad \mathcal{N}_{-}=\left\{u \in H_{0}^{1}(\Omega) \mid I(u)<0\right\} .
$$


Energy $d$ is characterized by

$$
d=\min _{u \in H_{0}^{1}(\Omega) \backslash\{0\}} \max _{s \geq 0} J(s u)=\min _{u \in \mathcal{N}} J(u) .
$$

We also need to consider the (open) sublevels of $J$ :

$$
J^{k}=\left\{u \in H_{0}^{1}(\Omega) \mid J(u)<k\right\} .
$$

Hence,

$$
\mathcal{N}_{\alpha}=\mathcal{N} \cap J^{\alpha} \equiv\left\{u \in \mathcal{N} \mid\|u\|_{H_{0}^{1}}<\sqrt{\frac{2 d(p+1)}{p-1}}\right\} \neq \varnothing \quad \text { for all } \alpha>d
$$

The above alternative characterization of $d$ also shows that

$$
\operatorname{dist}(0, \mathcal{N})=\min _{u \in \mathcal{N}}\|u\|_{H_{0}^{1}}=\delta:=\sqrt{\frac{2 d(p+1)}{p-1}}>0 .
$$

For all $\alpha>d$, we define

$$
\lambda_{\alpha}=\inf \left\{\|u\| \mid u \in \mathcal{N}_{\alpha}\right\}, \quad \Lambda_{\alpha}=\sup \left\{\|u\| \mid u \in \mathcal{N}_{\alpha}\right\} .
$$

We have the following monotonicity properties:

$$
\alpha \mapsto \lambda_{\alpha} \text { is nonincreasing, } \quad \alpha \mapsto \Lambda_{\alpha} \text { is nondecreasing. }
$$

In the following, we let $T^{*}\left(u_{0}\right)$ denote the maximal existence time of the solution with initial condition $u_{0} \in H_{0}^{1}(\Omega)$. We denote by $S(t)$ the nonlinear semigroup associated with problem (1.4)-(1.6). Instead of $u=u(t)$ we also write $S(t) u_{0}$ for $t<T^{*}\left(u_{0}\right)$. The smoothing properties of this semigroup suggest that we consider the space

$$
C_{0}^{1}(\Omega):=\left\{u \in C^{1}(\bar{\Omega}) \mid u=0 \text { on } \partial \Omega\right\}=C^{1}(\bar{\Omega}) \cap H_{0}^{1}(\Omega),
$$

endowed with the standard norm $\|\cdot\|_{C^{1}}$ of $C^{1}(\bar{\Omega})$. If $T^{*}\left(u_{0}\right)=\infty$, we denote by

$$
\omega\left(u_{0}\right):=\bigcap_{t \geq 0} \overline{\{u(s): s \geq t\}}
$$

the $\omega$-limit set of $u_{0} \in H_{0}^{1}(\Omega)$.

Let us introduce the sets

$$
\begin{aligned}
\mathcal{B} & =\left\{u_{0} \in H_{0}^{1}(\Omega) \mid \text { the solution } u=u(t) \text { of (1.4)-(1.6) blows up in finite time }\right\}, \\
\mathcal{G} & =\left\{u_{0} \in H_{0}^{1}(\Omega) \mid \text { the solution } u=u(t) \text { of (1.4)-(1.6) exists for all } t>0\right\}, \\
\mathcal{G} \circ & =\left\{u_{0} \in G \mid u(t) \mapsto 0 \text { in } H_{0}^{1}(\Omega) \text { as } t \rightarrow \infty\right\} .
\end{aligned}
$$


3.2. Stationary problem and comparison principle To obtain the comparison principle of problem (1.4)-(1.6), which is used to obtain the global existence and blowup of solutions for (1.4)-(1.6) with high initial energy $J\left(u_{0}\right)>d$, we need to consider the following stationary problem:

$$
\begin{aligned}
-\Delta u & \equiv f(u)=\sum_{k=1}^{l} a_{k}|u|^{p_{k}-1} u-\sum_{j=1}^{s} b_{j}|u|^{q_{j}-1} u \quad \text { in } \Omega, \\
u & =0 \quad \text { on } \partial \Omega .
\end{aligned}
$$

For problem (3.4)-(3.5), Simon [9] has proved the following lemma.

Lemma 3.1 [9]. Let $u_{0} \in H_{0}^{1}(\Omega)$ be such that $T^{*}\left(u_{0}\right)=\infty$. Then we have the convergence of solutions $S(t) u_{0}$ to the solution of problem (3.4)-(3.5).

Throughout this paper, it is important to prove the comparison principle of problem (1.4)-(1.6). To make extensive use of the following comparison principle for initial data $u_{0} \in H_{0}^{1}(\Omega)$, we first introduce some preliminary results (Lemma 3.2, Theorem 3.3 and Lemma 3.4).

Lemma 3.2 (Gronwall inequality). Let $y(t) \in L^{1}[0, T]$ and $y(0)=a$. If there exists $a$ constant $b$ such that $(d / d t) y(t) \leq b y(t)$, then $y(t) \leq a e^{b t}$.

Theorem 3.3 [8]. $T^{*}: H_{0}^{1}(\Omega) \rightarrow(0, \infty]$ is continuous. Moreover, for all $u_{0} \in H_{0}^{1}(\Omega)$ and for all $t \in\left(0, T^{*}\left(u_{0}\right)\right)$, the semigroup $S(t)$ maps an $H_{0}^{1}(\Omega)$ neighbourhood of $u_{0}$ continuously into $C_{0}^{1}(\Omega)$.

Lemma 3.4 (Strong maximum principle [1, p. 332]). Assume that

$$
u(x, t) \in C^{2,1}(\Omega \times(0, T)) \bigcap C(\bar{\Omega} \times(0, T)),
$$

where $\Omega$ is connected and bounded.

(i) If $u_{t}-\Delta u \leq 0$ and $u$ attains its maximum over $\bar{\Omega} \times(0, T)$ at a point $\left(x_{0}, t_{0}\right) \in$ $\Omega \times(0, T)$, then $u$ is constant on $t=t_{0}, x \in \Omega$.

(ii) If $u_{t}-\Delta u \geq 0$ and $u$ attains its minimum over $\bar{\Omega} \times(0, T)$ at a point $\left(x_{0}, t_{0}\right) \in$ $\Omega \times(0, T)$, then $u$ is constant on $t=t_{0}, x \in \Omega$.

We now state and prove the comparison principle of problem (1.4)-(1.6), which is applied to prove the global existence and nonexistence of solutions.

Lemma 3.5 (Comparison principle). Let $u_{0}, v_{0} \in H_{0}^{1}(\Omega)$ be such that $u_{0}-v_{0} \in \mathbb{K}$. Then $\left[S(t) u_{0}-S(t) v_{0}\right] \in \mathbb{K}$ for all $t \geq 0$. Moreover, if $u_{0} \neq v_{0}$ then, for $t>0$,

$$
S(t) u_{0}-S(t) v_{0}>0 \quad \text { in } \Omega .
$$

Proof. We put $u(t):=S(t) u_{0}, v(t):=S(t) v_{0}$. For all $T<\bar{T}:=\min \left\{T^{*}\left(u_{0}\right), T^{*}\left(v_{0}\right)\right\}$, let $\omega:=u-v$. We subtract the two equations for $u$ and $v$ given by (1.4). We have

$$
\omega_{t}-\Delta \omega=\sum_{k=1}^{l} a_{k}\left(|u|^{p_{k}-1} u-|v|^{p_{k}-1} v\right)-\sum_{j=1}^{s} b_{j}\left(|u|^{q_{j}-1} u-|v|^{q_{j}-1} v\right) \quad \text { in } \Omega \times(0, \bar{T}) \text {. }
$$


Use the mean value theorem, and for $\theta \in(0,1)$ assume that

$$
f(A)=\sum_{k=1}^{l} a_{k}|A|^{p_{k}-1} A-\sum_{j=1}^{s} b_{j}|A|^{q_{j}-1} A .
$$

Then

$$
\begin{aligned}
f\left(A_{1}\right)-f\left(A_{2}\right)= & \left(\sum_{k=1}^{l} a_{k} \int_{0}^{1} p_{k}\left|A_{1}+\theta\left(A_{1}-A_{2}\right)\right|^{p_{k}-1} d \theta\right)\left(A_{1}-A_{2}\right) \\
& -\left(\sum_{j=1}^{s} b_{j} \int_{0}^{1} q_{j}\left|A_{1}+\theta\left(A_{1}-A_{2}\right)\right|^{q_{j}-1} d \theta\right)\left(A_{1}-A_{2}\right) .
\end{aligned}
$$

Let

$$
\begin{aligned}
f\left(A_{1}\right) & =\sum_{k=1}^{l} a_{k}|u|^{p_{k}-1} u-\sum_{j=1}^{s} b_{j}|u|^{q_{j}-1} u, \\
f\left(A_{2}\right) & =\sum_{k=1}^{l} a_{k}|v|^{p_{k}-1} v-\sum_{j=1}^{s} b_{j}|v|^{q_{j}-1} v .
\end{aligned}
$$

Then

$$
\begin{aligned}
& \sum_{k=1}^{l} a_{k}\left(|u|^{p_{k}-1} u-|v|^{p_{k}-1} v\right)-\sum_{j=1}^{s} b_{j}\left(|u|^{q_{j}-1} u-|v|^{q_{j}-1} v\right) \\
& \quad=\left(\sum_{k=1}^{l} a_{k} \int_{0}^{1} p_{k}|u+\theta \omega|^{p_{k}-1} d \theta-\sum_{j=1}^{s} b_{j} \int_{0}^{1} q_{j}|u+\theta \omega|^{q_{j}-1} d \theta\right) \omega .
\end{aligned}
$$

For $x \in \Omega, t \geq 0$, let

$$
H(x, t)=\sum_{k=1}^{l} a_{k} p_{k} \int_{0}^{1}|u+\theta \omega|^{p_{k}-1} d \theta-\sum_{j=1}^{s} b_{j} q_{j} \int_{0}^{1}|u+\theta \omega|^{q_{j}-1} d \theta .
$$

Because $u, v$ are continuous functions, for all $T \in(0, \bar{T})$ we obtain

$$
M_{T}:=\sup _{\Omega \times(0, T)} H(x, t)<\infty .
$$

Then

$$
\begin{cases}\omega_{t}-\Delta \omega=H(t) \omega & \text { in } \Omega \times(0, \bar{T}), \\ \omega(0)=u_{0}-v_{0} \geq 0 & \text { in } \Omega, \\ \omega=0 & \text { on } \partial \Omega \times(0, \bar{T}) .\end{cases}
$$

If we multiply (3.6) by $\omega^{-}$and integrate on $\Omega$, then

$$
\frac{1}{2} \frac{d}{d t}\left\|\omega^{-}(t)\right\|^{2}=\int_{\Omega} H(t)\left|\omega^{-}(t)\right|^{2} d x-\left\|\nabla \omega^{-}(t)\right\|^{2} \leq M_{T}\left\|\omega^{-}(t)\right\|^{2}
$$


for all $t \in[0, T]$. By the Gronwall inequality and since $T$ is arbitrary, this proves that $\omega^{-}(t) \equiv 0$ and, in turn, the comparison principle for smooth initial data $u_{0}$ and $v_{0}$.

For $u_{0}, v_{0} \in H_{0}^{1}(\Omega)$, choose two sequences $\left\{u_{0}^{m}\right\},\left\{v_{0}^{m}\right\} \subset C_{c}^{\infty}(\Omega)$. For $m \rightarrow \infty$, we have $u_{0}^{m} \rightarrow u_{0}, v_{0}^{m} \rightarrow v_{0}$ in $H_{0}^{1}(\Omega), v_{0}^{m} \leq v_{0} \leq u_{0} \leq u_{0}^{m}$ in $\Omega$ for all $m$. If $v(X, T)>u(X, T)$ for some $(X, T) \in \Omega \times(0, \bar{T}), v^{m}=S(t) v_{0}^{m}, u^{m}=S(t) u_{0}^{m}$, by Theorem 3.3 we also have $v^{m}(X, T)>v(X, T)>u(X, T)>u^{m}(X, T)$ for sufficiently large $m$. Then $v_{0}^{m}>v_{0}>u_{0}>$ $u_{0}^{m}$. This contradicts the just proved comparison principle for smooth initial data. Then we have $\omega(t)=S(t) u_{0}-S(t) v_{0} \geq 0$ and $\left[S(t) u_{0}-S(t) v_{0}\right] \in \mathbb{K}$. Now we prove $H(t) \geq 0$. Arguing by contradiction, suppose that $H(t)<0$. Then $\omega_{t}-\Delta \omega=H(t) \omega \leq 0$. From Lemma 3.4 (the strong maximum principle), if $\omega_{t}-\Delta \omega \leq 0$ and $\omega$ attains its maximum over $\bar{\Omega} \times(0, T)$ at a point $\left(x_{0}, t_{0}\right) \in \Omega \times(0, T)$, then $\omega=c$. From the Dirichlet boundary condition $\left.\omega\right|_{\partial \Omega}=0$ we know that $\omega=0$, which contradicts the fact that $\omega$ is a nontrivial solution. Therefore, $H(t) \geq 0$. Since $\omega(t)=S(t) u_{0}-S(t) v_{0}$ satisfies the equation $\omega_{t}-\Delta \omega=H(t) \omega \geq 0$ together with a homogeneous Dirichlet boundary condition, the strong maximum principle implies that $\omega(t)>0$ in $\Omega$. The comparison principle is proved.

Lemma 3.6. If $u$ is a nontrivial solution of problem (3.4)-(3.5), then $J^{\prime \prime}(u)(u, u)<0$ and the first eigenvalue of the eigenvalue problem

$$
\begin{aligned}
-\Delta \psi-\sum_{k=1}^{l} a_{k} p_{k}|u|^{p_{k}-1} \psi+\sum_{j=1}^{s} b_{j} q_{j}|u|^{q_{j}-1} \psi & =\lambda \psi \quad \text { in } \Omega, \\
\psi & =0 \quad \text { on } \partial \Omega,
\end{aligned}
$$

is negative.

Proof. A nontrivial solution $u$ of problem (3.4)-(3.5) satisfies

$$
\|\nabla u\|^{2}=\sum_{k=1}^{l} a_{k}\|u\|_{p_{k}+1}^{p_{k}+1}-\sum_{j=1}^{s} b_{j}\|u\|_{q_{j}+1}^{q_{j}+1},
$$

which implies that $I(u)=0$. Hence

$$
\begin{aligned}
J^{\prime}(u) & =\lim _{\varepsilon \rightarrow 0} \frac{J((1+\varepsilon) u)-J(u)}{\varepsilon} \\
& =\|\nabla u\|^{2}-\sum_{k=1}^{l} a_{k}\|u\|_{p_{k}+1}^{p_{k}+1}+\sum_{j=1}^{s} b_{j}\|u\|_{q_{j}+1}^{q_{j}+1} \\
& =0, \\
J^{\prime \prime}(u) & =2 \lim _{\varepsilon \rightarrow 0} \frac{J((1+\varepsilon) u)-J(u)}{\varepsilon^{2}} \\
& =\|\nabla u\|^{2}-\sum_{k=1}^{l} a_{k} p_{k}\|u\|_{p_{k}+1}^{p_{k}+1}+\sum_{j=1}^{s} b_{j} q_{j}\|u\|_{q_{j}+1}^{q_{j}+1}
\end{aligned}
$$




$$
\begin{aligned}
& <\sum_{k=1}^{l} a_{k}\|u\|_{p_{k}+1}^{p_{k}+1}-\sum_{j=1}^{s} b_{j}\|u\|_{q_{j}+1}^{q_{j}+1}-\sum_{k=1}^{l} a_{k} p_{k}\|u\|_{p_{k}+1}^{p_{k}+1}+\sum_{j=1}^{s} b_{j} p_{k}\|u\|_{q_{j}+1}^{q_{j}+1} \\
& =\left(1-p_{k}\right) \sum_{k=1}^{l} a_{k}\|u\|_{p_{k}+1}^{p_{k}+1}-\left(1-p_{k}\right) \sum_{j=1}^{s} b_{j}\|u\|_{q_{j}+1}^{q_{j}+1} \\
& =\left(1-p_{k}\right)\|\nabla u\|_{2}^{2}<0 .
\end{aligned}
$$

Multiplying the first equation in problem (3.7) by $u$ and integrating the result,

$$
\|\nabla u\|^{2}-\sum_{k=1}^{l} a_{k} p_{k}\|u\|_{p_{k}+1}^{p_{k}+1}+\sum_{j=1}^{s} b_{j} q_{j}\|u\|_{q_{j}+1}^{q_{j}+1}=\lambda\|u\|^{2} .
$$

Consequently, the first eigenvalue of problem (3.7) is negative.

Theorem 3.7. Assume that $u_{1}, u_{2} \in H_{0}^{1}(\Omega) \backslash\{0\}$ solve problem (3.4)-(3.5) with $u_{1} \leq u_{2}$. Then either $u_{1}<0<u_{2}$ or $u_{1} \equiv u_{2}$.

Proof. Suppose that $u_{1} \not \equiv u_{2}$. Then, by comparison, we have $u_{1}<u_{2}$ in $\Omega$. By Lemma 3.6, the first eigenvalues $\lambda_{u_{1}}$ and $\lambda_{u_{2}}$ of the eigenvalue problems

$$
\begin{aligned}
-\Delta \psi-\sum_{k=1}^{l} a_{k} p_{k}\left|u_{i}\right|^{p_{k}-1} \psi+\sum_{j=1}^{s} b_{j} q_{j}\left|u_{i}\right|^{q_{j}-1} \psi=\lambda \psi & \text { in } \Omega, \\
\psi=0 & \text { on } \partial \Omega,
\end{aligned}
$$

$i=1,2$, are negative. Denote by $e_{1}$ and $e_{2}$, respectively, the corresponding positive first eigenfunctions. Then

$$
J^{\prime \prime}\left(u_{1}\right)\left(e_{1}, e_{1}\right)<0 \quad \text { and } \quad J^{\prime \prime}\left(u_{2}\right)\left(e_{2}, e_{2}\right)<0 .
$$

Since $J^{\prime \prime}$ is continuous,

$$
\begin{aligned}
& J\left(u_{1}+\delta e_{1}\right)=J\left(u_{1}\right)+\frac{\delta^{2}}{2} J^{\prime \prime}\left(u_{1}\right)\left(e_{1}, e_{1}\right)+o\left(\delta^{2}\right)<J\left(u_{1}\right), \\
& J\left(u_{2}-\delta e_{2}\right)=J\left(u_{2}\right)+\frac{\delta^{2}}{2} J^{\prime \prime}\left(u_{2}\right)\left(e_{2}, e_{2}\right)+o\left(\delta^{2}\right)<J\left(u_{2}\right)
\end{aligned}
$$

for sufficiently small $\delta>0$. Consider the closed set

$$
Q=\left\{w \in H_{0}^{1}(\Omega) \mid u_{1} \leq w \leq u_{2} \text { almost everywhere in } \Omega\right\} \subset H_{0}^{1}(\Omega)
$$

and put

$$
m:=\inf _{u \in Q} J(u)
$$

Since $u_{1}<u_{1}+\delta e_{1}<u_{2}-\delta e_{2}<u_{2}$ for small $\delta>0$, inequalities (3.8) and (3.9) imply that

$$
m<\min \left\{J\left(u_{1}\right), J\left(u_{2}\right)\right\}
$$


We claim that $m$ is achieved by a function $w \in Q$. Indeed, let $\left\{w_{n}\right\}_{n} \subset Q$ be a minimizing sequence for $\left.J\right|_{Q}$. Then

$$
\begin{aligned}
\left\|\nabla w_{n}\right\|^{2} & =2 J\left(w_{n}\right)+\sum_{k=1}^{l} \frac{2 a_{k}}{p_{k}+1}\left\|w_{n}\right\|_{p_{k}+1}^{p_{k}+1}-\sum_{j=1}^{s} \frac{2 b_{j}}{q_{j}+1}\left\|w_{n}\right\|_{q_{j}+1}^{q_{j}+1} \\
\leq & 2 J\left(w_{n}\right)+\sum_{k=1}^{l} \frac{2 a_{k}}{p_{k}+1}\left(\left\|u_{1}\right\|_{p_{k}+1}^{p_{k}+1}+\left\|u_{2}\right\|_{p_{k}+1}^{p_{k}+1}\right) \\
& \quad-\sum_{j=1}^{s} \frac{2 b_{j}}{q_{j}+1}\left(\left\|u_{1}\right\|_{q_{j}+1}^{q_{j}+1}+\left\|u_{2}\right\|_{q_{j}+1}^{q_{j}+1}\right) \\
\leq C &
\end{aligned}
$$

where $C>0$ is a constant independent of $n$. Passing to a subsequence, we have $w_{n} \rightarrow w \in H_{0}^{1}(\Omega)$ and

$$
\begin{aligned}
& w_{n} \rightarrow w \quad \text { almost everywhere in } \Omega, \\
& \left\|w_{n}\right\|_{p_{k}+1}^{p_{k}+1} \rightarrow\|w\|_{p_{k}+1}^{p_{k}+1}, \quad\left\|w_{n}\right\|_{q_{j}+1}^{q_{j}+1} \rightarrow\|w\|_{q_{j}+1}^{q_{j}+1}
\end{aligned}
$$

We conclude that $w \in Q$ and that

$$
\begin{aligned}
J(w) & =\frac{1}{2}\|\nabla w\|^{2}-\sum_{k=1}^{l} \frac{a_{k}}{p_{k}+1}\|w\|_{p_{k}+1}^{p_{k}+1}+\sum_{j=1}^{s} \frac{b_{j}}{q_{j}+1}\|w\|_{q_{j}+1}^{q_{j}+1} \\
& \leq \frac{1}{2} \liminf _{n \rightarrow \infty}\left\|\nabla w_{n}\right\|^{2}-\sum_{k=1}^{l} \frac{a_{k}}{p_{k}+1}\left\|w_{n}\right\|_{p_{k}+1}^{p_{k}+1}+\sum_{j=1}^{s} \frac{b_{j}}{q_{j}+1}\left\|w_{n}\right\|_{q_{j}+1}^{q_{j}+1} \\
& =\liminf _{n \rightarrow \infty} J\left(w_{n}\right)=m .
\end{aligned}
$$

This forces $J(w)=m$, so that $w$ is a minimizer for $\left.J\right|_{Q}$. By (3.10) we have $w \neq u_{1}$, $w \neq u_{2}$. Moreover, the comparison principle implies that $S(t) w \in Q$ and therefore $J(S(t) w) \geq m$ for all $t>0$. On the other hand,

$$
\frac{d}{d t} J(u)=-\left\|u_{t}\right\|^{2} \leq 0,
$$

so $J(S(t) w) \geq m$ for all $t>0$. These two facts enable us to conclude that $S(t) w=w$ for all $t>0$, which implies that $w$ is a solution of problem (3.4)-(3.5) and $u_{1}<w<u_{2}$. For $|\varepsilon|$ sufficiently small, we have $(1+\varepsilon) w \in Q$ such that the minimization property of $w$ yields

$$
J^{\prime \prime}(w)(w, w)=2 \lim _{t \rightarrow 0} \frac{J((1+\varepsilon) w)-J(w)}{\varepsilon^{2}} \geq 0 .
$$

By Lemma 3.6, this implies that $w \equiv 0$ and completes the proof.

REMARK 3.8. The result and proof of Theorem 3.7 can carry over to the case where $u_{1}$ is a subsolution and $u_{2}$ is a supersolution of problem (3.4)-(3.5). 
3.3. Probabilities of the existence and nonexistence of global solutions We now obtain a result on nontrivial solutions of problem (3.4)-(3.5), which plays a necessary role in obtaining the global existence and blow-up at high energy level.

Theorem 3.9. Let $u$ be a nontrivial solution of problem (3.4)-(3.5), and $u_{0} \in H_{0}^{1}(\Omega)$, $u_{0} \not \equiv u$.

(i) If $u^{+} \not \equiv 0$ and $u_{0} \geq u$, then $u_{0} \in \mathcal{B}$.

(ii) If $u^{-} \not \equiv 0$ and $u_{0} \leq u$, then $u_{0} \in \mathcal{B}$.

(iii) If $u>0$ and $u \geq u_{0} \geq-u$, then $u_{0} \in \mathcal{G}_{0}$.

Proof. (i) If $u_{0}$ is not in $\mathcal{B}$, then by Lemma 3.1 we have $s(t) u_{0} \rightarrow u^{\prime}$ as $t \rightarrow \infty$, where $u^{\prime}$ is a solution of problem (3.4)-(3.5). By comparison and since $u_{0} \not \equiv u$, we have $S(t) u_{0}>S(t) u$. Taking the limits of both sides, we obtain $u^{\prime}>u$. By Theorem 3.7 we have $u^{\prime}>0>u$, which contradicts $u^{+} \not \equiv 0$. Thus $u_{0} \in \mathcal{B}$. Part (ii) is proved in the same way.

(iii) Since $u \geq u_{0} \geq-u$, by comparison we have $u \in \mathcal{G}$. By Lemma 3.1, we have $s(t) u_{0} \rightarrow u^{\prime}$ as $t \rightarrow \infty$, where $u^{\prime}$ is a solution of problem (3.4)-(3.5). We also obtain $u>u^{\prime}>-u$ by comparison. If $u \neq \equiv$, by Theorem 3.7 we have $u>0>u^{\prime}$, which contradicts $u^{\prime}>0>-u$. Hence $u^{\prime}=0$, that is, $u_{0} \in \mathcal{G}_{0}$.

Lemma 3.10. Let $u_{0} \in H_{0}^{1}(\Omega)$ and put $u(t)=S(t) u_{0}$ for $t \in\left[0, T^{*}\left(u_{0}\right)\right)$. Then for all $t \in\left(0, T^{*}\left(u_{0}\right)\right)$

$$
\frac{d}{d t}\|u(t)\|^{2}=-2 I(u)
$$

Proof. Multiplying equation (1.4) by $u(t)$ and integrating the obtained result on $\Omega$,

$$
\int_{\Omega} u_{t} u d x-\int_{\Omega} \Delta u u d x=\sum_{k=1}^{l} a_{k} \int_{\Omega}|u|^{p_{k}-1} u u d x-\sum_{j=1}^{s} b_{j} \int_{\Omega}|u|^{q_{j}-1} u u d x .
$$

Equivalently,

$$
\frac{1}{2} \frac{d}{d t}\|u(t)\|^{2}=-\left(\|\nabla u(t)\|^{2}-\sum_{k=1}^{l} a_{k}\|u(t)\|_{p_{k}+1}^{p_{k}+1}+\sum_{j=1}^{s} b_{j}\|u(t)\|_{q_{j}+1}^{q_{j}+1}\right),
$$

which implies the result.

We see that Lemma 3.10 implies that, in a weak sense, equation (1.4) is dissipative in $\mathcal{N}_{+}$. However, the following result shows that initial data in $\mathcal{N}_{+}$with high energy may generate both vanishing solutions and solutions which blow up.

Theorem 3.11 (Global existence and blow-up at high energy level). For any $M>0$, there exist $u_{M}, v_{M} \in \mathcal{N}_{+} \cap \mathbb{K} \cap C_{0}^{1}(\Omega)$ with $J\left(u_{M}\right), J\left(v_{M}\right) \geq M$ and $u_{M} \in \mathcal{G}_{0}, v_{M} \in \mathcal{B}$.

Proof. Let $M>0$ and let $v$ denote a positive solution of problem (3.4)-(3.5). Let $c>0$ and let $\Omega^{\prime} \subset \Omega$ be an open subset such that $v>c$ on $\Omega^{\prime}$. For $k>0$, choose a positive function $\varphi_{k} \in C_{0}^{1}\left(\Omega^{\prime}\right)$ such that $\left\|\nabla \varphi_{k}\right\| \geq k$ and $\left\|\varphi_{k}\right\|_{\infty} \leq c$. 
Fix $k>0$ and put $\omega_{+}:=v+\varphi_{k}, \omega_{-}:=v-\varphi_{k}$. Then $\omega_{ \pm} \in \mathbb{K}$, and

$$
\begin{aligned}
& \left\|\nabla \omega_{ \pm}\right\| \geq\left\|\varphi_{k}\right\|-\|\nabla v\| \geq k-\|\nabla v\|, \\
& \left\|\omega_{ \pm}\right\|_{p_{k}+1} \leq\|v\|_{p_{k}+1}+\left\|\varphi_{k}\right\|_{p_{k}+1} \leq\|v\|_{p_{k}+1}+c\left|\Omega^{\prime}\right|^{1 /\left(p_{k}+1\right)} .
\end{aligned}
$$

Hence, if $k$ is sufficiently large,

$$
\begin{aligned}
J\left(\omega_{ \pm}\right) & =\frac{1}{2}\left\|\nabla \omega_{ \pm}\right\|^{2}-\sum_{k=1}^{l} \frac{a_{k}}{p_{k}+1}\left\|\omega_{ \pm}\right\|_{p_{k}+1}^{p_{k}+1}+\sum_{j=1}^{s} \frac{b_{j}}{q_{j}+1}\left\|\omega_{ \pm}\right\|_{q_{j}+1}^{q_{j}+1} \\
& \geq \frac{1}{2}\left\|\nabla \omega_{ \pm}\right\|^{2}-\sum_{k=1}^{l} \frac{a_{k}}{p_{k}+1}\left\|\omega_{ \pm}\right\|_{p_{k}+1}^{p_{k}+1} \\
& \geq \frac{1}{2}(k-\|\nabla v\|)^{2}-\sum_{k=1}^{l} \frac{a_{k}}{p_{k}+1}\left(\|v\|_{p_{k}+1}+c\left|\Omega^{\prime}\right|^{1 / p_{k}+1}\right)^{p_{k}+1}
\end{aligned}
$$

and

$$
\begin{aligned}
I\left(\omega_{ \pm}\right) & =\left\|\nabla \omega_{ \pm}\right\|^{2}-\sum_{k=1}^{l} a_{k}\left\|\omega_{ \pm}\right\|_{p_{k}+1}^{p_{k}+1}+\sum_{j=1}^{s} b_{j}\left\|\omega_{ \pm}\right\|_{q_{j}+1}^{q_{j}+1} \\
& \geq\left\|\nabla \omega_{ \pm}\right\|^{2}-\sum_{k=1}^{l} a_{k}\left\|\omega_{ \pm}\right\|_{p_{k}+1}^{p_{k}+1} \\
& >(k-\|\nabla v\|)^{2}-\sum_{k=1}^{l} a_{k}\left(\|v\|_{p_{k}+1}+c\left|\Omega^{\prime}\right|^{1 / p_{k}+1}\right)^{p_{k}+1} .
\end{aligned}
$$

For $k$ sufficiently large we have both $J\left(\omega_{ \pm}\right) \geq M$ and $I\left(\omega_{ \pm}\right)>0$, hence $\omega_{ \pm} \in \mathcal{N}_{+}$. For such a number $k$, take $u_{M}=\omega_{-}$and $v_{M}=\omega_{+}$. Because $0 \leq u_{M} \leq v$, we have $u_{M} \in \mathcal{G}_{0}$ by Theorem 3.9(iii). Because $v_{M} \geq v$, we also have $v_{M} \in \mathcal{B}$ by Theorem 3.9(i).

3.4. Sufficient conditions for global and nonglobal existence of solutions In order to acquire an abstract criterion for vanishing (respectively, blow-up) in terms of the variational values $\lambda_{\alpha}$ and $\Lambda_{\alpha}$ defined by (3.3), we need some lemmas.

Lemma 3.12 [7]. Let $u \in H_{0}^{1}(\Omega)$ and $\|\nabla u\| \neq 0$. Then:

(i) $\lim _{s \rightarrow 0} J(s u)=0$ and $\lim _{s \rightarrow+\infty} J(s u)=-\infty$;

(ii) on the interval $0<s<\infty$, there exists a unique $s^{*}=s^{*}(u)$ such that

$$
\left.\frac{d}{d s} J(s u)\right|_{s=s^{*}}=0 ;
$$

(iii) $J(s u)$ is increasing on $0 \leq s \leq s^{*}$, decreasing on $s^{*} \leq s<\infty$, and takes its maximum at $s=s^{*}$

(iv) $I(s u)>0$ for $0<s<s^{*}, I(s u)<0$ for $s^{*}<s<\infty$, and $I\left(s^{*} u\right)=0$. 
Lemma 3.13. $J(u)>0$ for any $u \in \mathcal{N}_{+}$. Moreover, for all $u \in \mathcal{N}$, we have $J(u)=$ $\max _{s \geq 0} J(s u)$. Finally, for any $k>0$, the set $J^{k} \cap \mathcal{N}_{+}$is bounded in $H_{0}^{1}(\Omega)$.

Proof. As in Lemma 3.12, for $s>0$,

$$
\begin{aligned}
J(s u) & =\frac{s^{2}}{2}\|\nabla u\|^{2}-\sum_{k=1}^{l} \frac{a_{k} s^{p_{k}+1}}{p_{k}+1}\|u\|_{p_{k}+1}^{p_{k}+1}+\sum_{j=1}^{s} \frac{b_{j} s^{q_{j}+1}}{q_{j}+1}\|u\|_{q_{j}+1}^{q_{j}+1}, \\
\frac{d}{d s} J(s u) & =s\|\nabla u\|^{2}-\sum_{k=1}^{l} a_{k} s^{p_{k}}\|u\|_{p_{k}+1}^{p_{k}+1}+\sum_{j=1}^{s} b_{j} s^{q_{j}}\|u\|_{q_{j}+1}^{q_{j}+1},
\end{aligned}
$$

and there exists an $s^{*}$ such that $\left.(d / d s) J(s u)\right|_{s=s^{*}}=0$. From equations (3.1) and (3.2), assumption (1.7) and since $u \in \mathcal{N}_{+}$,

$$
\begin{aligned}
J(u) & =\frac{1}{2}\|\nabla u\|^{2}-\sum_{k=1}^{l} \frac{a_{k}}{p_{k}+1}\|u\|_{p_{k}+1}^{p_{k}+1}+\sum_{j=1}^{s} \frac{b_{j}}{q_{j}+1}\|u\|_{q_{j}+1}^{q_{j}+1} \\
& >\frac{1}{p+1} I(u)+\frac{p-1}{2(p+1)}\|\nabla u\|^{2}>0 .
\end{aligned}
$$

For $u \in \mathcal{N}$, we obtain

$$
I(u)=\|\nabla u\|^{2}-\sum_{k=1}^{l} a_{k}\|u\|_{p_{k}+1}^{p_{k}+1}+\sum_{j=1}^{s} b_{j}\|u\|_{q_{j}+1}^{q_{j}+1}=0 .
$$

From Lemma 3.12(iv) we have $s^{*}=1$ and by Lemma 3.12(iii) this implies that $J(u)=\max _{s \geq 0} J(s u)$ for any $u \in \mathcal{N}$. From $J(u)<k$, inequality (3.12) and $I(u)>0$,

$$
\|\nabla u\|^{2}<\frac{2(p+1)}{p-1} k
$$

Therefore, for any $k>0$, the set $J^{k} \cap \mathcal{N}_{+}$is bounded in $H_{0}^{1}(\Omega)$.

We now give an abstract criterion for vanishing (respectively, blow-up) in terms of the variational values $\lambda_{\alpha}$ and $\Lambda_{\alpha}$ defined by (3.3).

Theorem 3.14. If $u_{0} \in \mathcal{N}_{+}$and $\left\|u_{0}\right\| \leq \lambda_{J\left(u_{0}\right)}$ then $u_{0} \in \mathcal{G}_{0}$. If $u_{0} \in \mathcal{N}_{-}$and $\left\|u_{0}\right\|_{2} \geq \Lambda_{J\left(u_{0}\right)}$ then $u_{0} \in \mathcal{B}$.

Proof. Let $u(t):=S(t) u_{0}$ for $t \in\left[0, T^{*}\left(u_{0}\right)\right)$. Multiplying equation (1.4) by $u_{t}(t)$ and integrating the obtained result on $\Omega$,

$$
\int_{\Omega} u_{t} u_{t} d x-\int_{\Omega} \Delta u u_{t} d x=\sum_{k=1}^{l} a_{k} \int_{\Omega}|u|^{p_{k}-1} u u_{t} d x-\sum_{j=1}^{s} b_{j} \int_{\Omega}|u|^{q_{j}-1} u u_{t} d x
$$

that is,

$$
\left\|u_{t}(t)\right\|^{2}+\frac{1}{2} \frac{d}{d t}\|\nabla u(t)\|^{2}=\frac{d}{d t} \sum_{k=1}^{l} \frac{a_{k}}{p_{k}+1}\|u(t)\|_{p_{k}+1}^{p_{k}+1}-\frac{d}{d t} \sum_{j=1}^{s} \frac{b_{j}}{q_{j}+1}\|u(t)\|_{q_{j}+1}^{q_{j}+1} .
$$


Equivalently,

$$
\frac{d}{d t} J(u)=-\left\|u_{t}(t)\right\|^{2}<0 .
$$

Noting inequality (3.13), we find that

$$
J^{\prime}\left(u_{0}\right) \neq 0 \quad \text { which implies } \quad J(u(t))<J\left(u_{0}\right) \text { for all } t \in\left(0, T^{*}\right) .
$$

Assume first that $u_{0} \in \mathcal{N}_{+}$satisfies $\left\|u_{0}\right\| \leq \lambda_{J\left(u_{0}\right)}$. We claim that $u(t) \in \mathcal{N}_{+}$for all $t \in\left[0, T^{*}\left(u_{0}\right)\right)$. By contradiction, if there exists $s>0$ such that $u(t) \in \mathcal{N}_{+}$for $0 \leq t<s$ and $u(s) \in \mathcal{N}$, then (3.11) and (3.14) imply that

$$
\|u(s)\|<\left\|u_{0}\right\| \leq \lambda_{J\left(u_{0}\right)}, \quad J(u(s))<J\left(u_{0}\right) .
$$

This contradicts the definition of $\lambda_{J\left(u_{0}\right)}$ and proves the claim. Hence, Lemma 3.13 shows that the orbit $\{u(t)\}$ remains bounded in $H_{0}^{1}(\Omega)$ for $t \in\left[0, T^{*}\left(u_{0}\right)\right)$, so that $T^{*}\left(u_{0}\right)=\infty$. Now for every $\omega \in \omega\left(u_{0}\right)$, by equation (3.11) and inequality (3.13) we obtain

$$
\|\omega\|<\lambda_{J\left(u_{0}\right)} \quad \text { and } \quad J(\omega) \leq J\left(u_{0}\right) .
$$

By the definition of $\lambda_{J\left(u_{0}\right)}, u_{0} \in \mathcal{N}_{+}$, and the definition of $\omega\left(u_{0}\right)$, we obtain $\omega\left(u_{0}\right) \subset \mathcal{N}_{+}$. Hence, we conclude that $\omega\left(u_{0}\right) \cap \mathcal{N}=\varnothing$. From the definition of $\mathcal{N}$ we know that $\omega\left(u_{0}\right)=\{0\}$. In other words, $u_{0} \in \mathcal{G}_{0}$, as claimed.

Assume now that $u_{0} \in \mathcal{N}_{-}$satisfies $\left\|u_{0}\right\| \geq \Lambda_{J\left(u_{0}\right)}$. A similar argument to that above shows that $u(t) \in \mathcal{N}_{-}$for all $t \in\left[0, T^{*}\left(u_{0}\right)\right)$. Now if, by contradiction, $T^{*}\left(u_{0}\right)=\infty$, then for every $\omega \in \omega\left(u_{0}\right)$ we would have by equation (3.11) and inequality (3.13),

$$
\|\omega\|>\Lambda_{J\left(u_{0}\right)} \quad \text { and } \quad J(\omega) \leq J\left(u_{0}\right) .
$$

By the definition of $\Lambda_{J\left(u_{0}\right)}$ we then infer that $\omega\left(u_{0}\right) \cap \mathcal{N}=\varnothing$. However, since $\operatorname{dist}\left(0, \mathcal{N}_{-}\right)>0$, we also have $0 \notin \omega\left(u_{0}\right)$. This gives $\omega\left(u_{0}\right)=\varnothing$, contrary to the assumption that $u(t)$ is a global solution. We conclude that $T^{*}\left(u_{0}\right)<\infty$, as claimed.

\section{Conclusion}

In this paper we considered the initial boundary value problems of the nonlinear wave equation and nonlinear heat equation. The two different equations share the same nonlinear terms and we derived results for high initial energy level for both equations. We point out that the results for the both equations are not parallel. At the high energy level, we only showed the blow-up results for the nonlinear wave equation by the potential well method. The conditions for this blow-up result are sufficient but not necessary, and we have no idea whether the solution will exist globally at the high energy level. For the heat equation, we discussed the probabilities of the existence and nonexistence of global solutions and gave some sufficient conditions for the global and nonglobal existence of solutions at high initial energy level by employing the comparison principle and variational methods. We derived the sufficient conditions not only for blow-up but also for global existence. This means that we know more about the heat equation than the wave equation. 


\section{Acknowledgements}

We thank the referee and the editor for their suggestion to add the Conclusion. This work was supported by the National Natural Science Foundation of China (11101102), Ph. D. Programs Foundation of Ministry of Education of China (20102304120022), Support Plan for the Young College Academic Backbone of Heilongjiang Province (1252G020), Natural Science Foundation of Heilongjiang Province (A201014), Science and Technology Research Project of Department of Education of Heilongjiang Province (12521401), and Foundational Science Foundation of Harbin Engineering University and Fundamental Research Funds for the Central Universities.

\section{References}

[1] L. C. Evans, Partial differential equations, volume 19 of Graduate Studies in Mathematics (American Mathematical Society, Providence, RI, 1998).

[2] F. Gazzola and M. Squassina, "Global solutions and finite time blow up for damped semilinear wave equations”, Annales de l'Institut Henri Poincaré (C) Analyse Non Linéaire 23 (2006) 185-207; doi:10.1016/j.anihpc.2005.02.007.

[3] F. Gazzola and T. Weth, "Finite time blow-up and global solutions for semilinear parabolic equations with initial data at high energy level", Differential Integral Equations 18 (2005) 961-990; http://projecteuclid.org/euclid.die/1356060117.

[4] K. Li and Q. Zhang, "Existence and nonexistence of global solutions for the equation of dislocation of crystals", J. Differential Equations 146 (1998) 5-21; doi:10.1006/jdeq.1998.3409.

[5] H. A. Levine, "Instability and nonexistence of global solutions to nonlinear wave equation of the form $P u_{t t}=-A u+\mathcal{F}(u)$ ", Trans. Amer. Math. Soc. 192 (1974) 1-21; doi:10.1090/S0002-9947-1974-0344697-2.

[6] H. A. Levine, "Some additional remarks on the nonexistence of global solutions to nonlinear wave equations", SIAM J. Math. Anal. 5 (1974) 138-146; doi:10.1137/0505015.

[7] Y. C. Liu and R. Z. Xu, "Wave equations and reaction-diffusion equations with several nonlinear source terms of different sign”, Discrete Contin. Dyn. Syst. Ser. B 7 (2007) 171-189; doi:10.3934/dcdsb.2007.7.171.

[8] P. Quittner, "Continuity of the blow-up time and a priori bounds for solutions in superlinear parabolic problems", Houston J. Math. 29 (2003) 757-799.

[9] L. Simon, "Asymptotics for a class of nonlinear evolution equations, with applications to geometric problems", Ann. of Math. (2) 118 (1983) 525-571; http://www.jstor.org/stable/2006981.

[10] T. Tao, M. Visan and X. Zhang, "The nonlinear Schrödinger equation with combined power-type nonlinearities", Comm. Partial Differential Equations 32 (2007) 1281-1343; doi:10.1080/03605300701588805.

[11] P. A. Vuillermot, "Small divisors and the construction of stable manifolds for nonlinear KleinGordon equations on $R_{0}^{+} \times R$ ", in: Nonlinear hyperbolic equations and field theory (eds M. K. Venkatesha Murthy and S. Spagnolo), (Longman Scientific \& Technical, Harlow, 1992), 197-213.

[12] T. Yu, L. Q. Tang, B. W. Liu and R. Z. Xu, "Wave equations and reaction-diffusion equations with several nonlinear source terms with critical energy", AIP Conf. Proc. 1479 (2012) 2435-2438; doi:10.1063/1.4756687. 